\title{
A Novel Hybrid Membrane VAD as First Step Toward Hemocompatible Blood Propulsion
}

\author{
Aldo Ferrari, ${ }^{1,3,4}$ Costanza Giampietro, ${ }^{1,3}$ Buörn Bachmann, ${ }^{1}$ \\ Laura Bernardi, ${ }^{4}$ Deon Bezuidenhhout, ${ }^{5}$ Paolo Ermanni, ${ }^{6}$ \\ Raoul Hopf, ${ }^{3,4}$ Sarah Kitz, ${ }^{9}$ Gerald Kress, ${ }^{6}$ Christian Loosli, ${ }^{6}$ \\ Vita Marina, ${ }^{4}$ Mirko Meboldt, ${ }^{7}$ Giovanni Pellegrini, ${ }^{9}$ \\ Dimos Poulikakos, ${ }^{1}$ Mathias Rebholz ${ }^{7}$ Marianne Schmid Daners (i), ${ }^{7}$ \\ Tanja Schmidt, ${ }^{8}$ Christoph Starck, ${ }^{2}$ Georgios Stefopoulos, ${ }^{1}$ \\ Simon Sündermann, ${ }^{10,2,11}$ Bente Thamsen, ${ }^{7}$ Peter Zilla, ${ }^{5}$ \\ Evgenij Potapov, ${ }^{2,10,11,12}$ Volkmar Falk, ${ }^{2,10,11,12}$ \\ and EDOARDO MAZZA 3,4
}

\begin{abstract}
${ }^{1}$ Laboratory of Thermodynamics in Emerging Technologies, Department of Mechanical and Process Engineering, ETH Zurich, Sonneggstrasse 3, 8092 Zurich, Switzerland; ${ }^{2}$ Department of Cardiothoracic and Vascular Surgery, German Heart Institute Berlin, Berlin, Germany; ${ }^{3}$ EMPA, Swiss Federal Laboratories for Material Science and Technologies, Überlandstrasse 129, 8600 Dübendorf, Switzerland; ${ }^{4}$ Institute for Mechanical Systems, ETH Zurich, 8092 Zurich, Switzerland; ${ }^{5}$ Christiaan Barnard Division of Cardiothoracic Surgery, University of Cape Town, Cape Town, South Africa; ${ }^{6}$ Laboratory of Composite Materials and Adaptive Structures, ETH Zurich, 8092 Zurich, Switzerland; ${ }^{7}$ Product Development Group Zurich, Department of Mechanical and Process Engineering, ETH Zurich, Zurich, Switzerland; ${ }^{8}$ Julius Wolff Institute and Center for Musculoskeletal Surgery, Charité - Universitätsmedizin, Berlin, Germany; ${ }^{9}$ Laboratory for Animal Model Pathology, Institute of Veterinary Pathology, Vetsuisse Faculty, University of Zurich, Zurich, Switzerland; ${ }^{10}$ Department of Cardiovascular Surgery, Charité Universitätsmedizin Berlin, Berlin, Germany; ${ }^{11}$ Deutsches Zentrum für Herz-Kreislaufforschung, Standort Berlin, Germany; and

${ }^{12}$ Translational Cardiovascular Technologies, Institute of Translational Medicine, Department of Health Sciences and Technology, Swiss Federal Institute of Technology (ETH), Zurich, Switzerland
\end{abstract}

(Received 26 February 2020; accepted 11 August 2020; published online 8 September 2020)

Associate Editor Tingrui Pan oversaw the review of this article.

\begin{abstract}
Heart failure is a raising cause of mortality. Heart transplantation and ventricular assist device (VAD) support represent the only available lifelines for end stage disease. In the context of donor organ shortage, the future role of VAD as destination therapy is emerging. Yet, major drawbacks are connected to the long-term implantation of current devices. Poor VAD hemocompatibility exposes the patient to life-
\end{abstract}

Address correspondence to Aldo Ferrari, Laboratory of Thermodynamics in Emerging Technologies, Department of Mechanical and Process Engineering, ETH Zurich, Sonneggstrasse 3, 8092 Zurich, Switzerland. Electronic mail: aldo.ferrari@empa. chVolkmar Falk, Department of Cardiothoracic and Vascular Surgery, German Heart Institute Berlin, Berlin, Germany. Electronic mail: falk@dhzb.deEdoardo Mazza, EMPA, Swiss Federal Laboratories for Material Science and Technologies, Überlandstrasse 129, 8600 Dübendorf, Switzerland. Electronic mail: mazza@imes.mavt. ethz.ch

Aldo Ferrari and Costanza Giampietro have contributed equally for this work. threatening events, including haemorrhagic syndromes and thrombosis. Here, we introduce a new concept of artificial support, the Hybrid Membrane VAD, as a first-of-its-kind pump prototype enabling physiological blood propulsion through the cyclic actuation of a hyperelastic membrane, enabling the protection from the thrombogenic interaction between blood and the implant materials. The centre of the luminal membrane surface displays a rationally-developed surface topography interfering with flow to support a living endothelium. The precast cell layer survives to a range of dynamically changing pump actuating conditions i.e., actuation frequency from 1 to $4 \mathrm{~Hz}$, stroke volume from 12 to $30 \mathrm{~mL}$, and support duration up to $313 \mathrm{~min}$, which are tested both in vitro and in vivo, ensuring the full retention of tissue integrity and connectivity under challenging conditions. In summary, the presented results constitute a proof of principle for the Hybrid Membrane VAD concept and represent the basis for its future development towards clinical validation.

Keywords-VAD, Endothelialization, Wall shear stress, Wall deformation, Hyperelastic membrane. 


\section{INTRODUCTION}

Continuous flow left ventricular assist devices (VADs) represent a routine treatment option for patients in end stage heart failure. In the first 2 years after implantation, the current generation of continuous flow VADs achieves survival comparable to heart transplantation. ${ }^{21,33}$ Survival and functional status are markedly improved when compared to medical therapy alone. ${ }^{38}$ On the other side, the generation of supraphysiological and turbulent flow within continuous flow VADs, and the concomitant interaction between blood and the thrombogenic materials at the device's luminal interface, expose the patients to a life-long risk of thromboembolic events and non-surgical bleeding. ${ }^{27,37,39}$ At the same time, patients with continuous flow pumps show a pathologic decrease of von Willebrand Factor (vWF) originating from an imbalance between the increased degradation induced by high wall shear stress (WSS) and the decreased endothelial release due to the absence of pulsatility. $5,7,8,31,32,40$

The preservation of pulsatility represents a target of future device development. ${ }^{40}$ Patients supported with the CARMAT total artificial heart, a pulsatile device partially covered with bovine pericardium at its luminal surface, showed reduced thromboembolic complications without the need for anticoagulation, highlighting the importance of a hemocompatible interface. ${ }^{17}$ The generation of surface textures at the luminal interface of HeartMate XVE supported the formation of a protective biological layer upon patient implantation $^{12}$ and reduced the risk of thromboembolic events without the need of anticoagulation therapy. ${ }^{35}$ However, the overall large pump size, its nonphysiological actuation and the uncontrolled generation of a biological layer represent limitations to be addressed.

Full, long-term hemocompatibility can only be bestowed by the combined protection from blood surface interactions inducing thrombosis and from blood damage, caused by non-physiological hemodynamic conditions created by the pump operation.

Here, we address the risk of thrombogenic events triggered by implant materials through the generation of a complete layer of living endothelial cells. ${ }^{6,23,34}$ In fact, a mature endothelium at the luminal interface of devices that are in contact with blood can provide protection from thrombus formation and subsequent adverse events. ${ }^{10}$

The endothelialization of blood pumps remains however largely unexplored as a result of two independent factors. First, the relative recent surge of pumping devices as destination therapy. In this novel, long-term perspective the interaction between implant materials and blood becomes a matter of concern to- wards the patient safety and life quality. Second, the non-physiological hemodynamic conditions created by the active implant, as compared to the limited interference created by passive elements such as vascular grafts. In this sense, endothelialization technologies applied to VADs face completely new challenges, which include the formation and/or maintenance of a connected tissue under supraphysiological combinations of WSS and wall deformation (WD). ${ }^{4}$ As most VAD-associated thrombotic events are registered during the first years of support an immediate protection must be considered. For this reason, the in vitro generation of a mature endothelial monolayer prior to device implantation must be preferred to ensure the luminal sheltering during the early phases of device actuation.

The actuation of blood pumps yields complex and dynamic gradients of flow-generated WSS which is accompanied by local strain (i.e., WD) when deformable elements are part of the actuation scheme. ${ }^{14}$ In particular, the luminal surface of current VAD features regions of perturbed hemodynamic loads, which prove non-viable for endothelial cells. ${ }^{1,36}$ This scenario requires a novel concept to be developed which shall include two converging approaches: (i) The establishment of an alternative pump design, engineered to support a failing heart while minimizing the hemodynamic load and therefore rendering a viable substrate for endothelialization ${ }^{2}$ and, (ii) The implementation of engineered luminal substrates, extending the survival of endothelial cells under highly dynamic and turbulent flow conditions. ${ }^{4}$

Here, we describe the development and validation (in vitro and in vivo) of a novel pump concept (the Hybrid Membrane VAD; HyMem-VAD) fulfilling the above design criteria. The primary objectives of the presented acute animal study were (i) the development and clinical adaptation of an endothelialization protocol, (ii) the test of safety and actuation performance for the HyMem-VAD, and (iii) the endpoint analysis of cell monolayer integrity upon several hours of pump actuation.

\section{MATERIALS AND METHODS}

\section{Membrane Fabrication}

The hybrid membrane was generated by molding the hyperelastic RTV 4420 silicone $^{11}$ into an elliptic layer. The chosen design parameters included an aspect ratio of 2:1 and length of the major radius of $44 \mathrm{~mm}$. The membrane features a pattern of corrugations in the cross section protruding along the minor axis (Fig. 1a). The specific design was optimized to reduce 
(a)

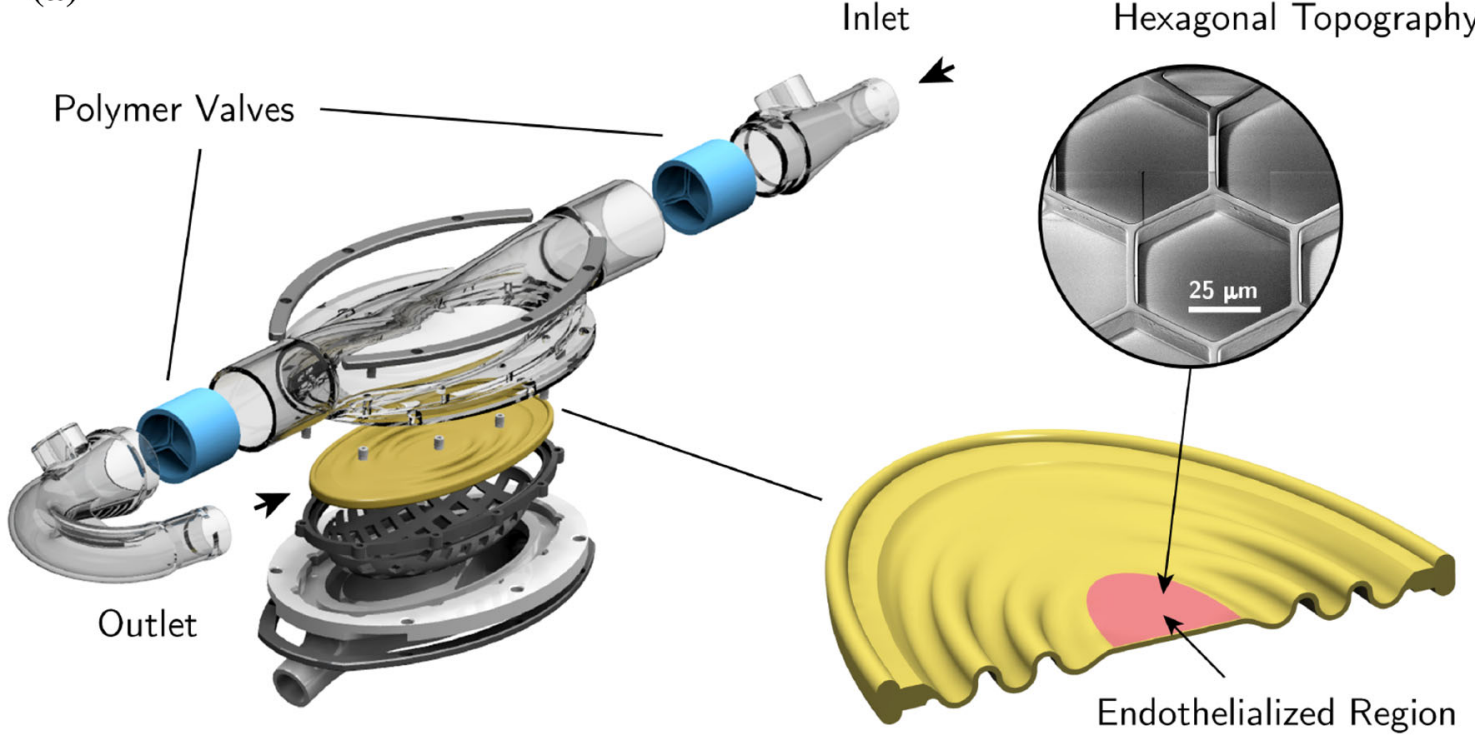

(b)

(c)

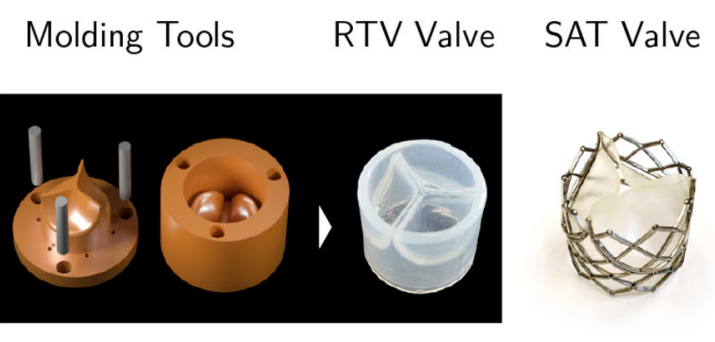

(d)

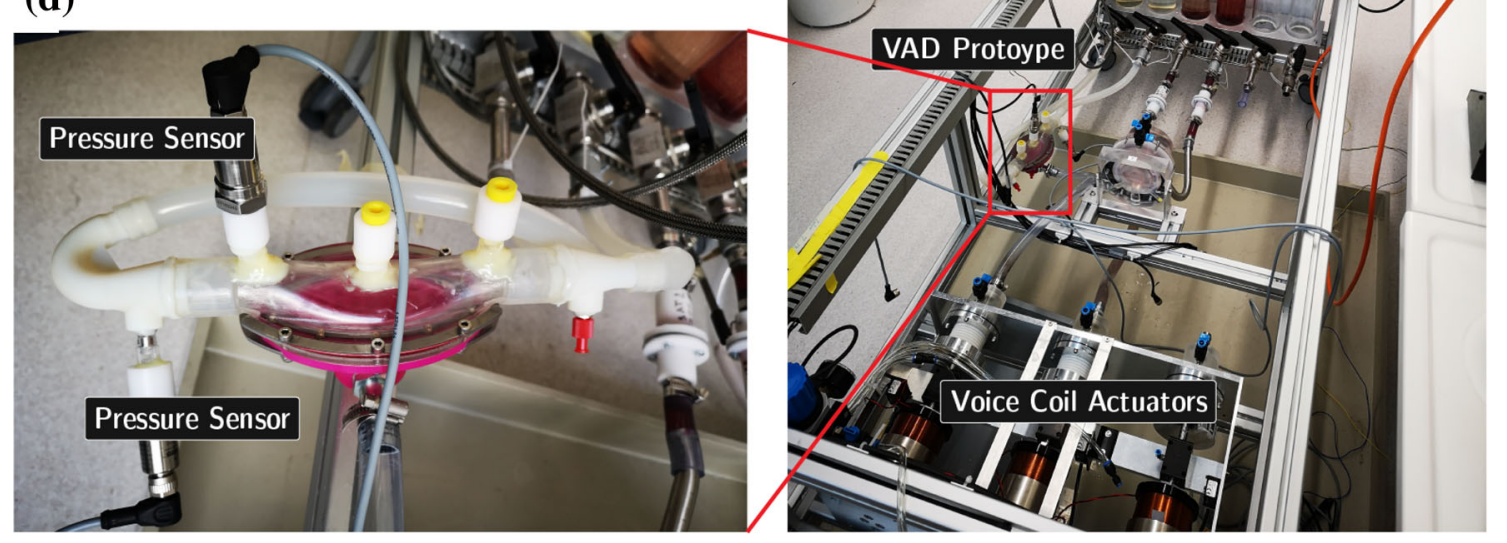

FIGURE 1. Hybrid Membrane VAD design. (a) The left-hand side shows the full pump assembly, including polymer valves (light blue) and the hybrid membrane (yellow). The right-hand side shows a close up section of the corrugated membrane. Red marks the endothelialized region, which contains the hexagonal surface topography (shown in SEM close-up). Panel (b): The parameterized design of molding tools and the resulting RTV polymer valve are shown on the left-hand side. On the right-hand side a TAVI valve (by SAT) is shown. Panel (c) shows the set-up of the long term test bench. The VAD is connected to vertical pressure cylinders with compliant silicone tubing. A magnification of the pump can be seen in panel (d). This version of the pump is fitted with additional pressure detection locations on the housing, allowing the measurement of pressure gradient across the valves. 
the extent of WD generated on the surface upon full membrane extrusion. ${ }^{19}$ In addition, the corrugations are tapered along the major axis to minimize their interference with flow. Upon actuation, the central region of the membrane is cyclically exposed to high levels of WSS (up to $15 \mathrm{~Pa}$ ) and WD (up to 16\% areal strain range), see Refs. 18 and 20. This demanding interface was therefore selected as benchmark to test endothelialization performance. Here, to locally support the adhesion of endothelial cells and the formation and maintenance of a connected endothelial monolayer, honeycomb hexagonal wells with optimized size and geometry (well basal surface $=1045 \mu \mathrm{m}^{2}, \quad$ well $\quad$ height $=6 \mu \mathrm{m}, \quad$ wall width $=4 \mu \mathrm{m}$ ) were implemented upon membrane fabrication (Fig. 1a ${ }^{4}$ ). Extended description is provided as supplementary information.

\section{Pump Design}

The HyMem-VAD is a pulsatile, volume displacement pump with design and operation features enabling actuation at high pulsation rates and thus minimizing the overall device volume. The technological core of the pumping scheme is represented by a hyperelastic silicone membrane which is cyclically actuated. The membrane features a pattern of luminal corrugations designed to mitigate the levels of tensile strain upon its full extension. Importantly, the pump design, operation and dedicated topographical patterning ${ }^{4}$ enable luminal endothelialization. We described the most relevant aspects of the in vitro pump development. ${ }^{3,11,24,29,36}$

Specifically, the pump structure comprises two superimposed chambers horizontally separated by the hybrid membrane (Figs. 1a, 1d). Blood flows in the upper chamber (the blood chamber) while a physiologic saline solution is injected into the lower chamber (actuation chamber). The flow directionality is imposed by one-way valves at the inlet and outlet of the blood chamber (Fig. 1a). In the actuation scheme, a cyclic volume exchange in the actuation chamber induces the controlled extrusion of the hybrid membrane, thus enabling blood propulsion. The hybrid membrane represents therefore the core technological element of this scheme. Extended description of the hybrid membrane fabrication is provided in "Materials and Methods" section.

The pump dimensions were set to yield at a maximum output of $5 \mathrm{l} / \mathrm{min}$ while maintaining the apex strain (i.e., at the central region) on the hybrid membrane below $6 \%$. To reduce the necessary stroke volume (SV) and the resulting overall pump size, the operating frequency was raised to $240 \mathrm{bpm}\left(4 \mathrm{~Hz}^{29}\right)$. The prototype features in- and outlet polymeric tran- scatheter valves (Figs. 1a and 1b). Two different valve types were tested: a TAVI valve (Strait Access Technologies, SAT, Cape Town, South Africa) and a custom-developed polymeric tri-leaflet valve (Fig. 1b), made from same silicone elastomer as the propulsion membrane and the coating of the housing (i.e., RTV 4420). The TAVI prosthesis consists of three polyurethane based leaflets, which are connected to a stainless steel stent. The custom made RTV valves were realized based on iterative design and manufactured using a 3D printed negative mold. Finally, the diameter of the chosen cannulae is $20 \mathrm{~mm}$ to mitigate WSS by reducing the resulting flow speeds.

\section{Computational Studies}

To evaluate the mechanical and fluid-dynamic loads generated by the pump actuation on the luminal surface of the hybrid membrane, a finite-element and a computational fluid dynamic (CFD) models were developed. The finite-element model rendered the expected membrane deformation, which constituted the input for CFD. This analysis did not consider fluidstructure interactions as for the present hemodynamic conditions no relevant coupling is expected.

The flow conditions in the pump were analyzed for different operating conditions in Refs. 18 and 20 and compared with those of a conventional circularly shaped pump chamber indicating improved conditions of WSS for the present elliptical design. Figure 2 shows examples of streamlines obtained from the integration of the corresponding velocity fields during the diastolic phase. For the present study, the analysis of simulation's results focused on ellipsoidal area with radius of $5 \mathrm{~mm}$, located at the center of the hybrid membrane (Fig. 1).

The numerical analysis was applied to estimate mechanical strain and fluid stress during pump operation. ${ }^{20}$ Particle tracking velocimetry (PTV) measurements were performed to validate the predicted velocity fields (Fig. 3) as previously reported. ${ }^{18-20}$

The WSS at the contacting surface was investigated by performing a non-steady state CFD study of the pump at two operation frequencies of $120 \mathrm{bpm}$ and $240 \mathrm{bpm}$ with a stroke volume of $30 \mathrm{~mL} .^{20}$ The results of these calculations, along with those of corresponding finite element simulations of the membrane deformation (see Supplementary Methods) were used to select the portion of the membrane to be covered with endothelial cells. The initial membrane shape was assumed to be flat in order to reduce the complexity of the CFD model. The membrane motion was implemented as a moving boundary into the finite-volume solver ANSYS Fluent 18.2 (ANSYS, “ANSYS Fluent 

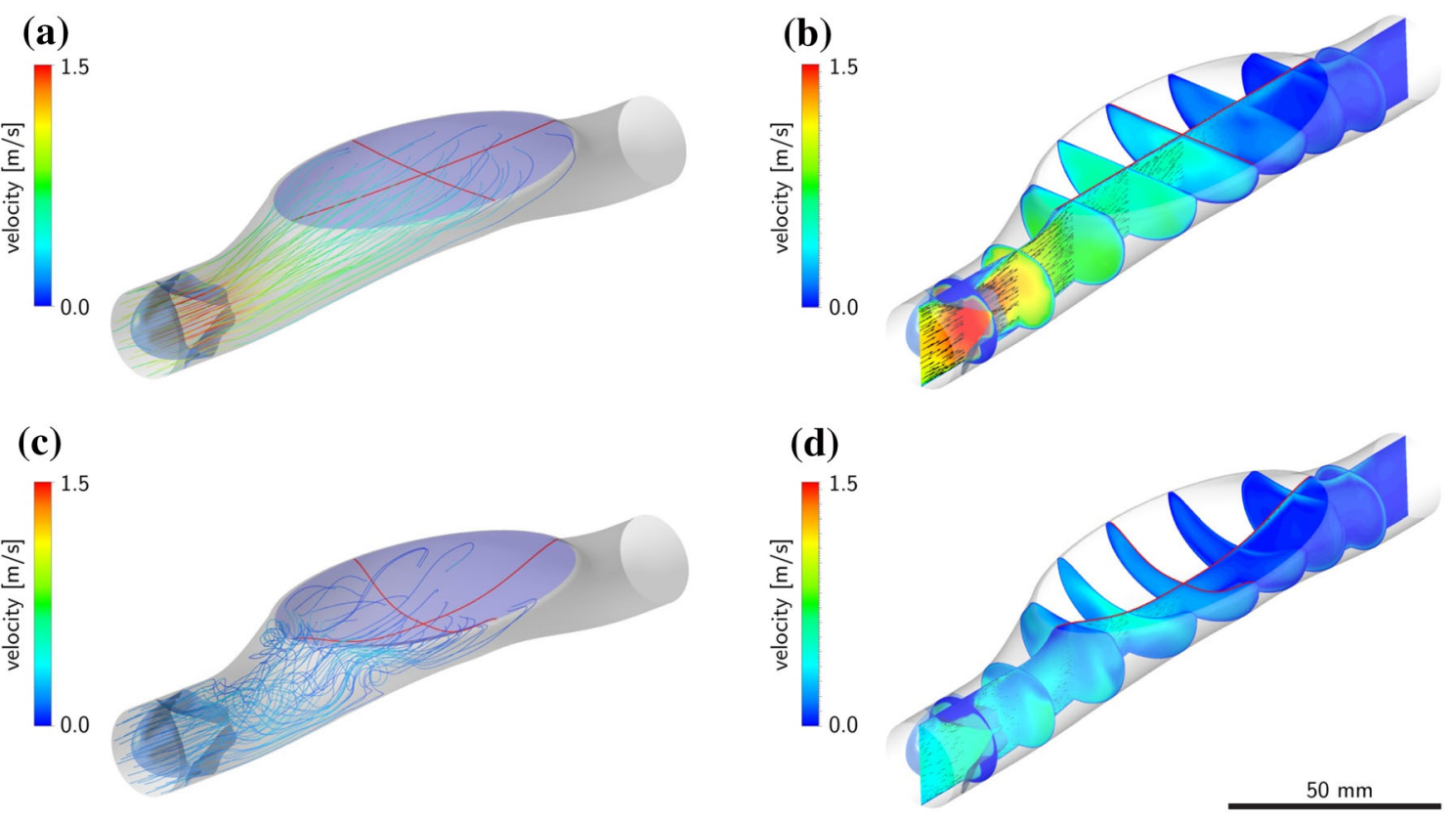

FIGURE 2. Illustration of the flow field: at the beginning ( $a$ and $b$ ) and at an intermediate state (c and d) of the diastolic phase. The images show the streamlines (a and $c$ ) for selected points at the inlet of the pump obtained from an integration of the corresponding velocity field (b and $d)$.

18.2 User's Guide." 2017). Extended description is provided as supplementary information.

\section{Long-Term In Vitro Testing}

The long term, cyclic behavior of the hybrid membrane was investigated using a custom-developed long term test bench (Figs. 1c and 1d). This test bench was designed to operate the pump at different actuation frequencies and SV under physiological pressure conditions. Various studies were performed with the whole pump exposed to a range of realistic operating conditions. It could be shown that a long term actuation does not impair the mechanical integrity of the silicone membrane. At $3 \mathrm{~L} / \mathrm{min}$ pumping rate (realized at $4 \mathrm{~Hz}$ actuation frequency and $12.5 \mathrm{~mL} \mathrm{SV}$ ), over 25 million cycles were achieved over a period of 2.5 months. Subsequently, the membrane was retrieved from the pump and visually inspected. No crack initiation sites could be found and also no zones of macroscopic plastic deformation could be identified. The endurance demonstrated in this test is in line with previous investigations on the cyclic behavior of the RTV 4420 silicone used for the hybrid membrane. ${ }^{11,14}$ These test results helped defining a safe-range for the actuation settings which were later used in the animal experiments. In order to demonstrate adequate endurance for clinical use, future investigations will involve long term tests with several hundred millions of cycles.

\section{Cell Culture}

Adult ovine endothelial cells (ECs) were harvested from fresh saphenous vein biopsies (Figs. $4 a$ and $4 b$ ). They were grown in medium 200PRF (\#M200PRF500, ThermoFisher Scientific) containing LSGS Kit (\#S-003-K, ThermoFisher Scientific). The kit includes fetal bovine serum (FBS) $2 \% \mathrm{v} / \mathrm{v}$; hydrocortisone $(1 \mu \mathrm{g} / \mathrm{mL})$; human epidermal growth factor $(10 \mathrm{ng} / \mathrm{mL})$; basic fibroblast growth factor $(3 \mathrm{ng} / \mathrm{mL})$ and heparin $(10 \mu \mathrm{g} / \mathrm{mL})$. Cells were maintained at $37{ }^{\circ} \mathrm{C}$ and $5 \% \quad \mathrm{CO}_{2}$. All reported in vivo experiments were performed using cells with less than 5 passages in vitro. Cells were tested for their ability to reconstitute a mature, growth-arrested endothelial monolayer on a synthetic surface. For this analysis, the target substrate was coated with cross-linked gelatin. ${ }^{16}$ Cells were seeded at high initial density $\left(10^{5}\right.$ cell $\left./ \mathrm{cm}^{2}\right)$.

Tissue generation was assessed after 3 days, through immunofluorescence analysis for endothelial junctional complexes, including VE-cadherin and $\beta$ catenin, major components of adherens junctions $\left(\mathrm{AJs}^{9}\right)$, and $\mathrm{ZO}-1$, major component of the tight junctions $\left(\mathrm{TJs}^{9}\right)$. The distribution of cell-to-cell junctions confirmed the generation of fully mature and connected endothelium on target substrates (Fig. 4c). Extended description is provided as supplementary information. 


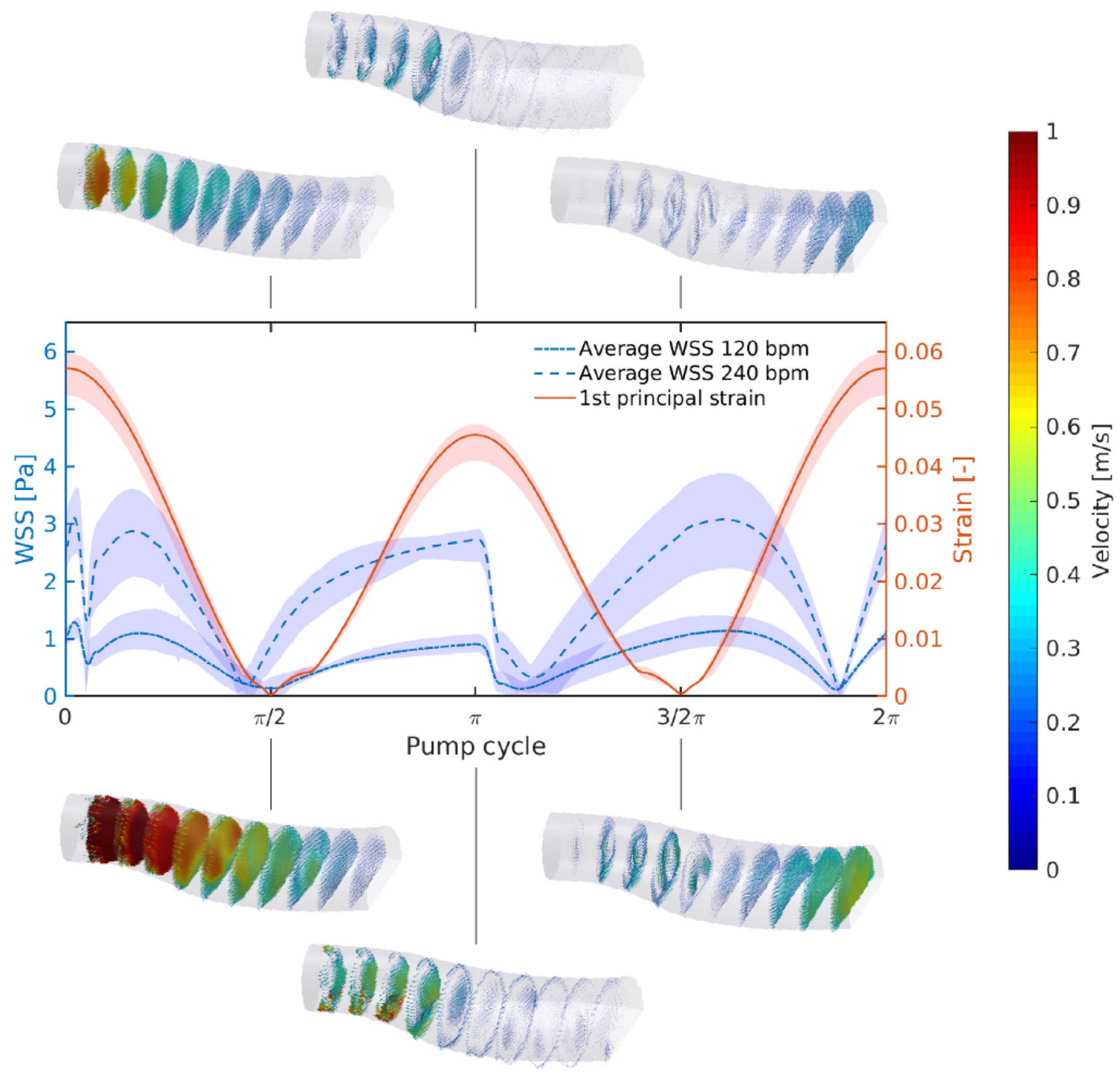

FIGURE 3. First Principal Elastic Strain and Wall Shear Stress at the cell contacting surface over one pump cycle for a pump frequency of 120 and $240 \mathrm{bpm}$. Time 0 corresponds to the beginning of the diastole. Sections of the vector-fields from PTV measurements are visualized above (corresponding to $120 \mathrm{bpm}$ ) and below (corresponding to $240 \mathrm{bpm}$ ). The color-bar corresponding to the measured flow velocity is given on the right-hand side.

\section{Antibodies}

The following primary antibodies were used: goat polyclonal anti-VE-cadherin (1:200, sc-6458, Santa Cruz Biotechnology Inc.), mouse monoclonal anti- $\beta$ catenin (1:100, \#610154, BD Biosciences), mouse monoclonal ZO-1 Alexa Fluor 555 (1:100, 1A12, ThermoFisher Scientific). F-actin was stained with TRIC-Phalloidin (SIGMA ALDRICH, P1951). Secondary antibodies were donkey anti-goat-Alexa 488 (A11055, Invitrogen), chicken anti-mouse-Alexa 647 (A21463, Invitrogen).

\section{Microscopy and Image Processing}

Fluorescent images of immunostained samples were acquired with a $\times 40,1.3 \mathrm{NA}$ oil immersion objective (Plan Fluor, Nikon, Japan), using a FITC filter, a TRITC filter, a Cy 5 filter and a DAPI filter for the cell nuclei. Only adjustment of brightness and contrast were used in the preparation of the figures. For comparison purposes, different sample images of the same antigen were acquired under the same microscopy acquisition settings. 
(a)

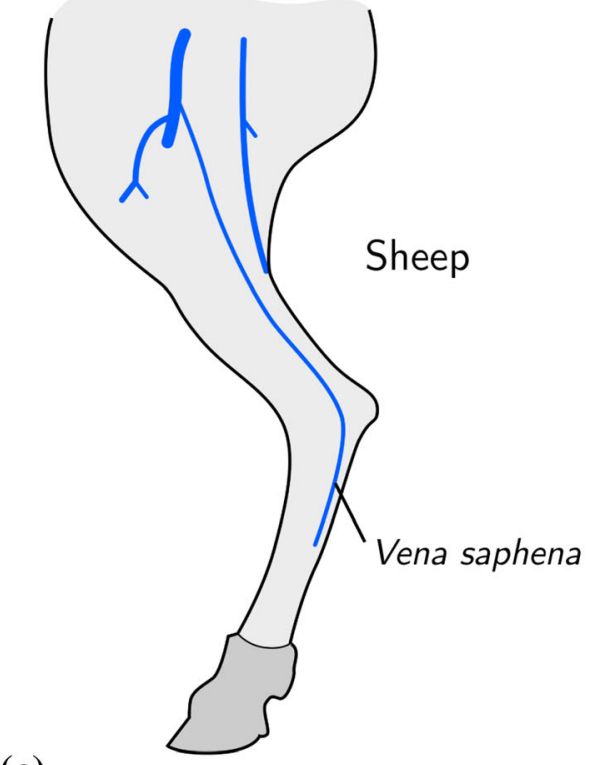

(b)

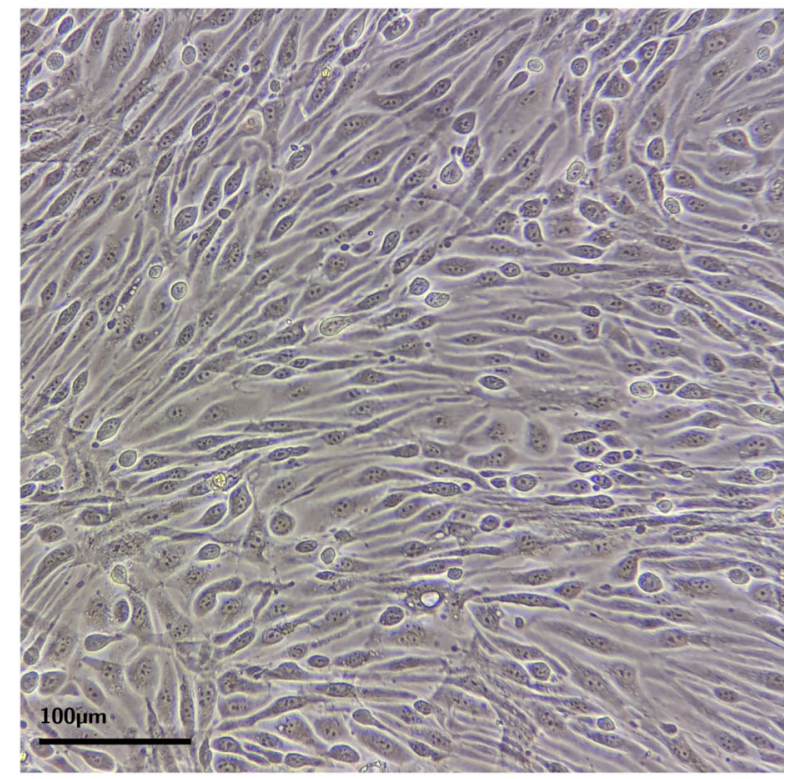

(c)
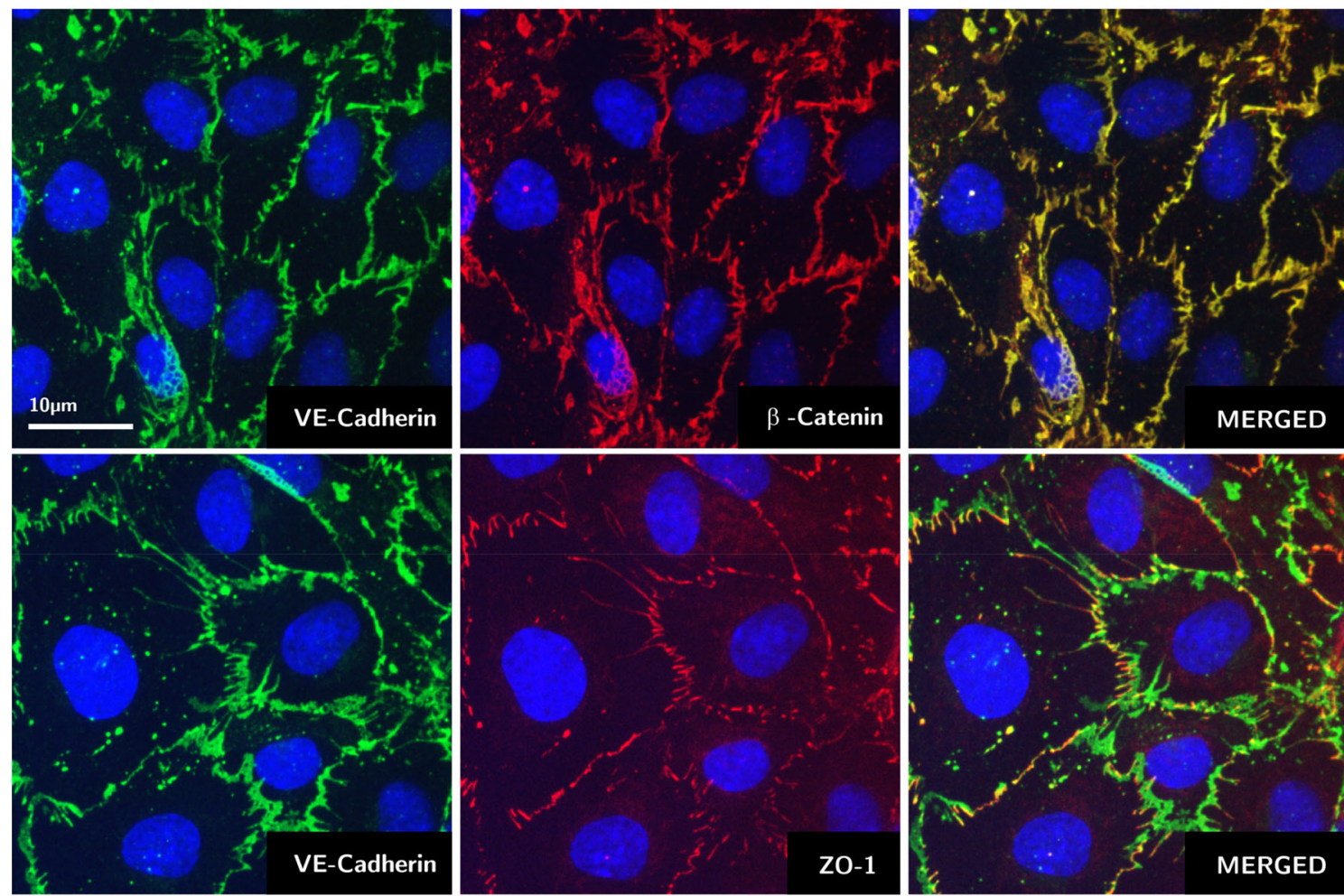

FIGURE 4. Isolation and characterization of ovine ECs. (a) Representation of the ovine Saphenous vein and (b) bright field image of a typical endothelial monolayer. Scale bar: $100 \mu \mathrm{m}$. (c) VE-cadherin, $\beta$-catenin and ZO-1 immunostaining of endothelial monolayers. Nuclei are stained with DAPI (blue). Scale bar: $10 \mu \mathrm{m}$.

\section{In Vivo Actuation}

All experimental protocols were approved by the Landesamt für Gesundheit und Soziales Berlin (LaGeSo, G 205/17), the local German authorities
(Number G 0215/17). All methods were carried out in accordance with relevant guidelines and regulations.

The actuation side of the pump housing was connected via a hydraulic tube to a PTFE bellows (ElringKlinger AG, Dettingen/Erms, Germany), which in 
TABLE 1. Overview of pump actuation parameters and their respective application durations

\begin{tabular}{|c|c|c|c|c|c|c|c|c|}
\hline \multicolumn{3}{|l|}{ Animal 2} & \multicolumn{3}{|c|}{ Animal 3} & \multicolumn{3}{|c|}{ Animal 4} \\
\hline $\begin{array}{l}\text { Frequency } \\
(\mathrm{bpm})\end{array}$ & $\begin{array}{l}\text { Volume } \\
\text { (mL) }\end{array}$ & $\begin{array}{l}\text { Duration } \\
\text { (min) }\end{array}$ & $\begin{array}{c}\text { Frequency } \\
\text { (bpm) }\end{array}$ & $\begin{array}{c}\text { Volume } \\
\text { (mL) }\end{array}$ & $\begin{array}{l}\text { Duration } \\
\text { (min) }\end{array}$ & $\begin{array}{c}\text { Frequency } \\
\text { (bpm) }\end{array}$ & $\begin{array}{c}\text { Volume } \\
\text { (mL) }\end{array}$ & $\begin{array}{c}\text { Duration } \\
\text { (min) }\end{array}$ \\
\hline 60 & 20 & 2 & 60 & 12 & 0 & 60 & 15 & 1 \\
\hline 60 & 30 & 31 & 80 & 20 & 1 & 120 & 12.5 & 10 \\
\hline 90 & 20 & 1 & 80 & 30 & 0 & 120 & 15 & 20 \\
\hline 90 & 30 & 15 & 90 & 30 & 313 & 120 & 20 & 12 \\
\hline 120 & 30 & 17 & & & & 180 & 15 & 0 \\
\hline 150 & 20 & 1 & & & & 240 & 12.5 & 0 \\
\hline 160 & 20 & 1 & & & & 240 & 15 & 53 \\
\hline 180 & 20 & 15 & & & & & & \\
\hline 180 & 30 & 4 & & & & & & \\
\hline 200 & 30 & 6 & & & & & & \\
\hline 240 & 15 & 7 & & & & & & \\
\hline
\end{tabular}

All times were rounded to the next minute. The pump implanted in animal 2 contained no cells.

turn was connected to a voice coil (lvcm-095-089-01, Moticont, Van Nuys, CA, USA) that compresses and stretches the bellows. Figure 8 shows a schematic of the actuator. An air bearing (S301301, Newway Air Bearings, Aston, PA, USA) kept the movement of the bellows and voice coil frictionless. As hydraulic fluid a saline solution was used which can be assumed to be incompressible. The stiff hydraulic line additionally allowed neglecting differences in displaced volume between bellows and membrane. The displacement volume of the membrane therefore could be directly correlated to the displacement of the bellows. For the trial the bellows and therefore the membrane were displaced in a sinusoidal way. The actuation parameters, the stroke volume and frequency together with their respective application times are shown in Table 1. By that, the cells were exposed to a broad range of operating points that covers the majority of flow conditions seen in patient application. These experimental conditions were selected for two main purposes: (i) to evaluate endothelial integrity over a wide range of pump operation states and (ii) to verify that the pump can be stably controlled over a wide range of operating conditions. The actuation sequence was selected to create rapid variations of local hemodynamic loads at the luminal surface of the hybrid membrane, therefore challenging the survival of endothelial cells. Extended description is provided as Supplementary Information.

\section{Sensors}

During the trial, the displacement of the membrane was measured by the bellow displacement with a laser sensor (OptoNCDT 1320, Micro-Epsilon Messtechnik $\mathrm{GmbH} \&$ Co. KG, Ortenburg, Germany) with a sample frequency of $2 \mathrm{kHz}$. The blood flow through the pump was measured by an ultrasonic flow sensor
(SONOFLOW CO.55, SONOTEC Ultraschallsensorik Halle $\mathrm{GmbH}$, Halle, Germany) placed around the outlet graft. Left ventricular, arterial and central venous pressure were recorded through TruWave pressure sensors (Edwards Lifesciences Corp., Irvine, USA). All data was sampled through a MF634 Humusoft Data Acquisition card (Humusoft s.r.o, Prague, Czech Republic) with a sampling frequency of $100 \mathrm{~Hz}$. The data acquisition was running simultaneously to the actuation control on a Real-Time Windows Target environment in MATLAB/Simulink (MathWorks, Inc., Natick, MA, USA).

\section{Statistical Analysis}

Statistical comparison of cell density was performed using a $t$ test. The Shapiro-Wilk test was used to test for normality of data with a significance level of 0.05 . The $p$-values reported in Fig. 5 are obtained by comparing several individual measures of cell density obtained in different fields of view for each individual sample. The resulting measure of density variation across the endothelial monolayer is relevant, as the initial phases of denudation (reporting on the failure of the endothelialization strategy and the maintenance of a fully confluent protection) appear with local, restricted loss of cell coverage.

\section{RESULTS}

\section{In Vitro Endothelialization}

The in vitro test bench was initially exploited to evaluate the performance of the proposed endothelialization approach. The conditions of pump actuation for these preparatory experiments fully reproduced those envisioned for the animal tests. ${ }^{20}$ The 
(a)

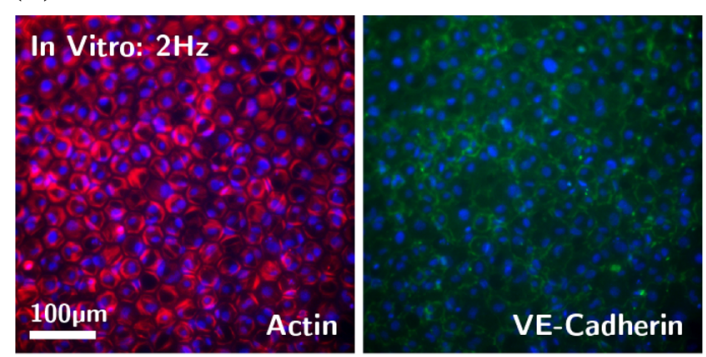

(b)

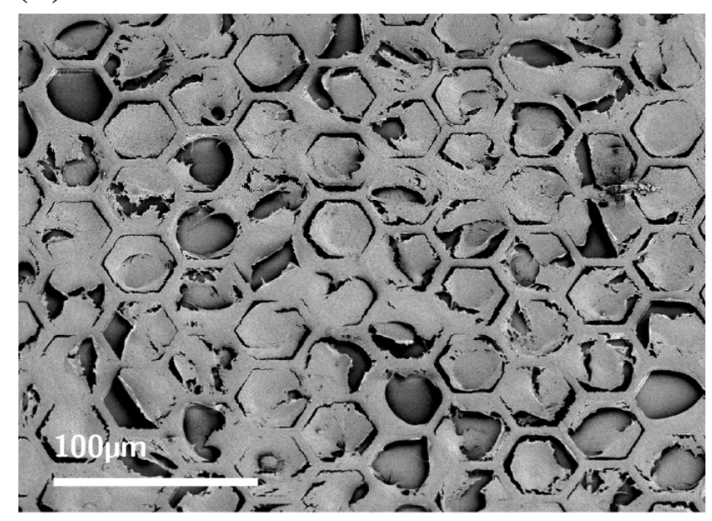

(c)

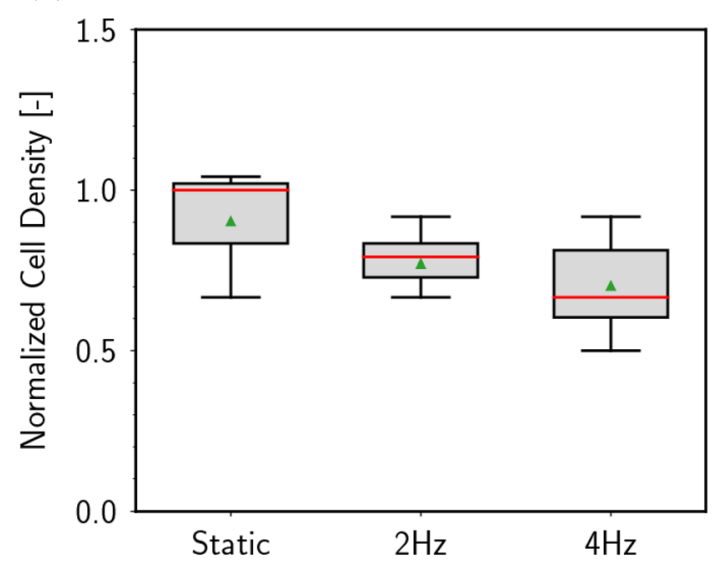

(d)
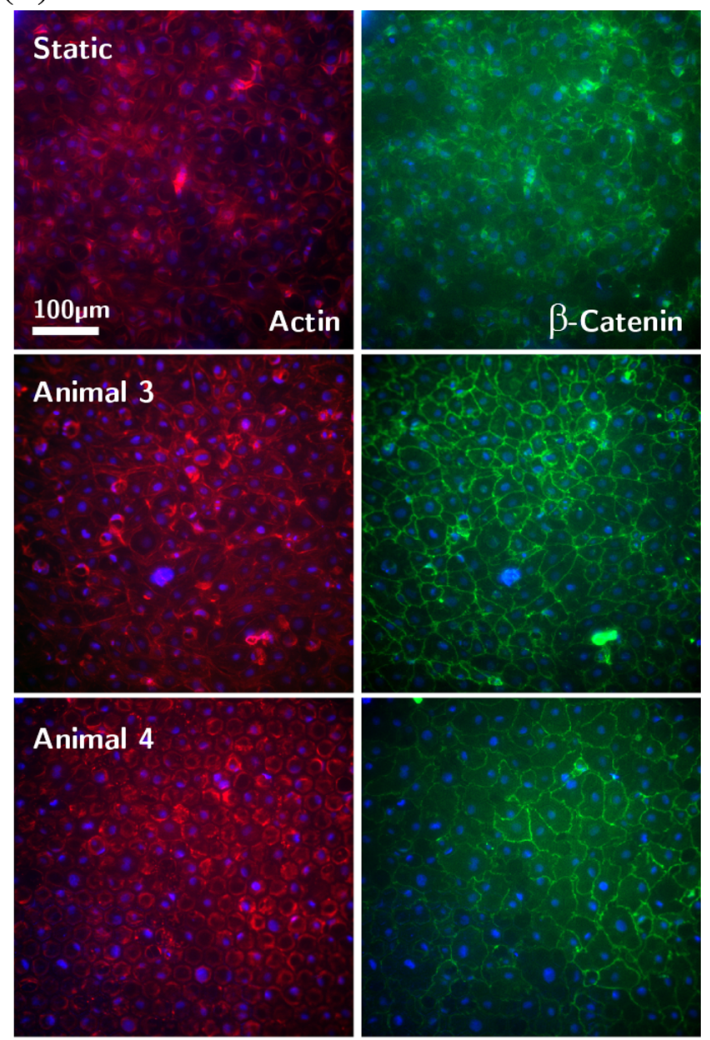

(e)

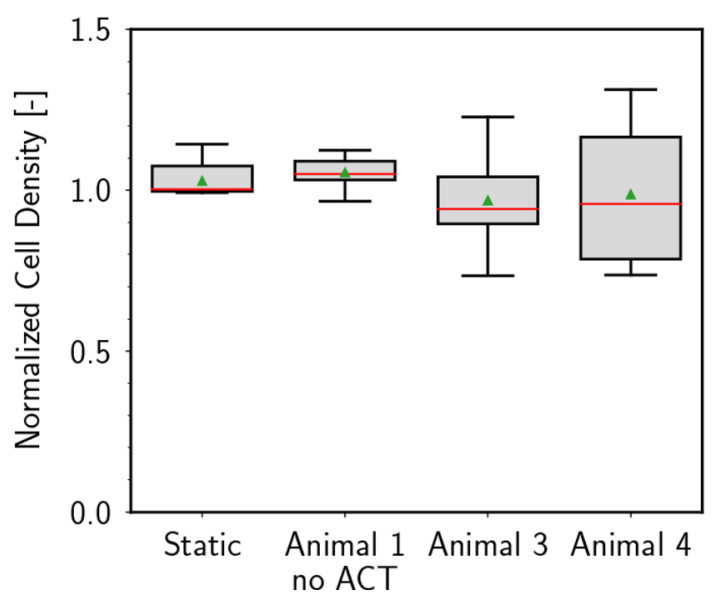

FIGURE 5. Analysis of endothelialization. In vitro test bench: (a) immunofluorescence analysis for actin (red) and VE-cadherin (green). Nuclei are stained with DAPI (blue). Scale bar: $100 \mu \mathrm{m}$; (b) SEM analysis of endothelialized hexagonal topography, scale bar: $100 \mu \mathrm{m}$, and (c) quantification of the cell density; no statistical significance: $2 \mathrm{~Hz}$ vs. static $p=0.4,4 \mathrm{~Hz}$ vs. static $p=0.2$. In vivo trials: (d) immunofluorescence analysis for actin (red) and $\beta$-catenin (green). Nuclei are stained with DAPI (blue). Scale bar: $100 \mu \mathrm{m},(\mathrm{e})$ quantification of the cell density; no statistical significance. Animal 1 vs. static $p=0.6$, Animal 3 vs. static $p=0.4$, Animal 4 vs. static $p=0.7$. Static samples were obtained maintaining the fully mounted device in static conditions in an incubator. These membranes did not undergo the process of implantation. On the other hand, the sample denoted as 'no actuation' (Animal 1) was fully implanted but not actuated. In this case the cells interacted with blood and were exposed to flow generated by the animal circulation.

central region of the hybrid membrane, featuring honeycomb hexagonal wells (Fig. 1a), was previously coated and seeded with ovine ECs. The selected topographical features support endothelial cell reten- tion without compromising monolayer integrity. ${ }^{4}$ The effect is obtained through a mitigation of the apparent WSS at the apical side of ECs nested in the hexagonal wells, as previously demonstrated by in vitro tests un- 
der a range of supraphysiological actuating conditions. ${ }^{4}$ In these tests, the topographically-modified surfaces were compared to an equivalent substrate featuring a completely flat surface and demonstrated a superior maintenance of monolayer integrity upon exposure to supraphysiological hemodynamic conditions of combined WSS and WD. ${ }^{4}$

After 3 days of incubation in static conditions, the endothelialized membrane was mounted in the hybrid membrane pump. During the entire procedure the luminal surface was maintained in contact with culture medium and care was taken to avoid bubbles. The prototype was then plugged to the test bench (Fig. 1c) and actuated while the entire device was maintained in an incubated environment. Specifically, the system was subjected to cyclic actuation at 2 or $4 \mathrm{~Hz}$, yielding an average output of $2.5 \mathrm{~L} / \mathrm{min}$ and $4.0 \mathrm{~L} / \mathrm{min}$, respectively. Cell survival and endothelialization performance were assessed through an endpoint immunostaining analysis for junctional proteins and actin cytoskeleton (Fig. 5a). Under all tested conditions, the luminal endothelium remained intact. Endothelial cell-to-cell junctions displayed the typical linear morphology along the boundaries between adjacent cells ${ }^{22}$ and were in contact with cortical Factin. Importantly, the cell morphology adapted to the underlying hexagonal topography, as previously reported (Fig. $5 b^{4}$ ). The resulting local cell density was comparable to the control (an identical membrane maintained in static conditions, Fig. 5c). In all, these results demonstrate that the endothelialization protocol and the actuation conditions within the HyMemVAD (see Materials and Methods) are viable and allow for the generation and maintenance of a connected endothelial monolayer. These achievements represented the prerequisite required to advance the endothelialization protocols to their application in animal trials.

\section{In Vivo Actuation}

All experimental animals (Animal \#1-4) were subjected to full implantation of the HyMem-VAD (Fig. 6). A complete description of the surgical settings is provided in Supplementary Materials and Methods. All trials were completed successfully. They allowed for the evaluation of the endothelialization procedure and of different actuation schemes, as previously tested in vitro (Figs. 4, 5, 6, 7, and 8).

Specifically, in Animal \#1 the pump was endothelialized but not actuated representing therefore a passive element in the circulation. This first test was necessary as internal control for the viability of the endothelialization and mounting procedures, as well as to assess the interaction between cells and blood. Animal \#2 served instead as control to minimize the risk of cell damage during the procedure of pump deployment, blood filling, connection with sensors and control system and initial actuation. The second main objective of this experiment was to evaluate the effectiveness of the control system of the pump in vivo. To this end, the animal physiological parameters (arterial blood pressure, oxygenation level and heart rate) were monitored for different pump actuation conditions and for the transient states between them. This allowed to verify that the interaction of our pump with the native organ was not causing detrimental effects at the given set-points and during the transition from an operating condition to the next one. The results were used to optimize the experimental procedures and the endothelialization approach in the ensuing animals. In Animals \#3 and 4 the HyMem-VAD was endothelial-
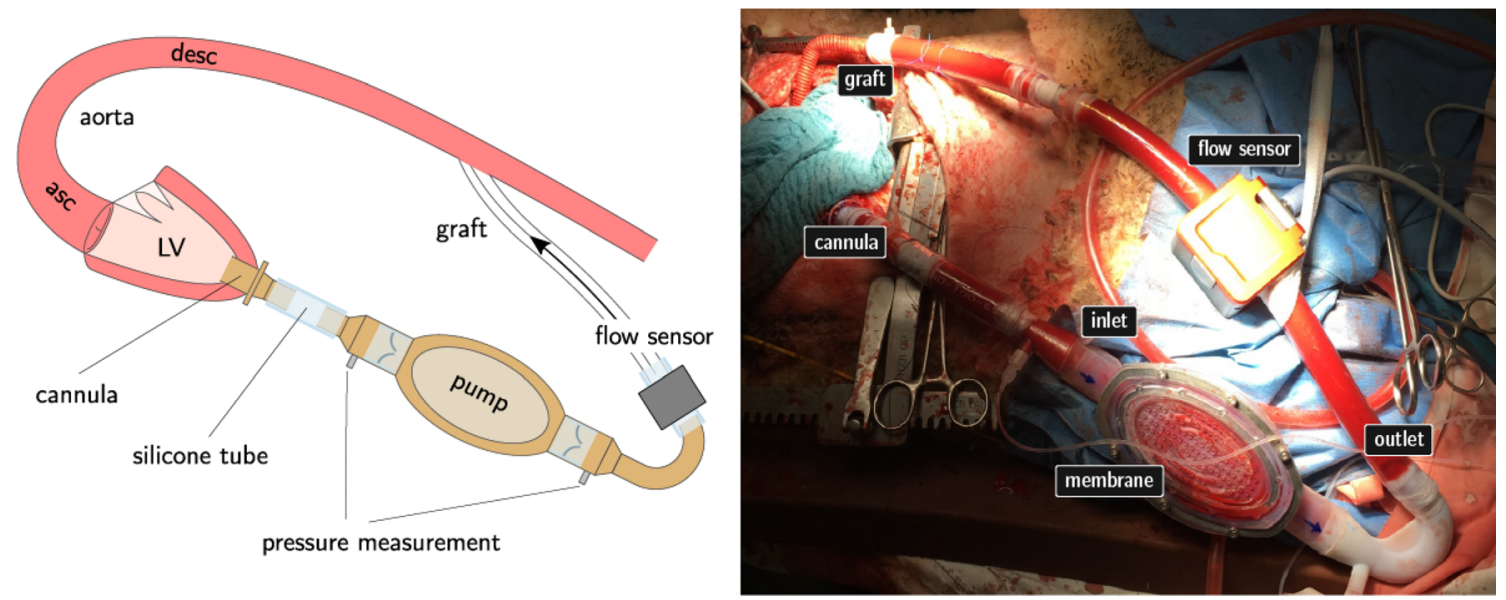

FIGURE 6. Experimental configuration of in vivo trials. Left: schematic representation of the pump configuration including pump housing with polymer valves at in- and outlet position. The inlet of the device is connected to the apex of the left ventricle. The graft on the outlet side connects to the descending aorta. Right: corresponding in vivo representation of the set-up during the trial. 
(a)

Animal 3
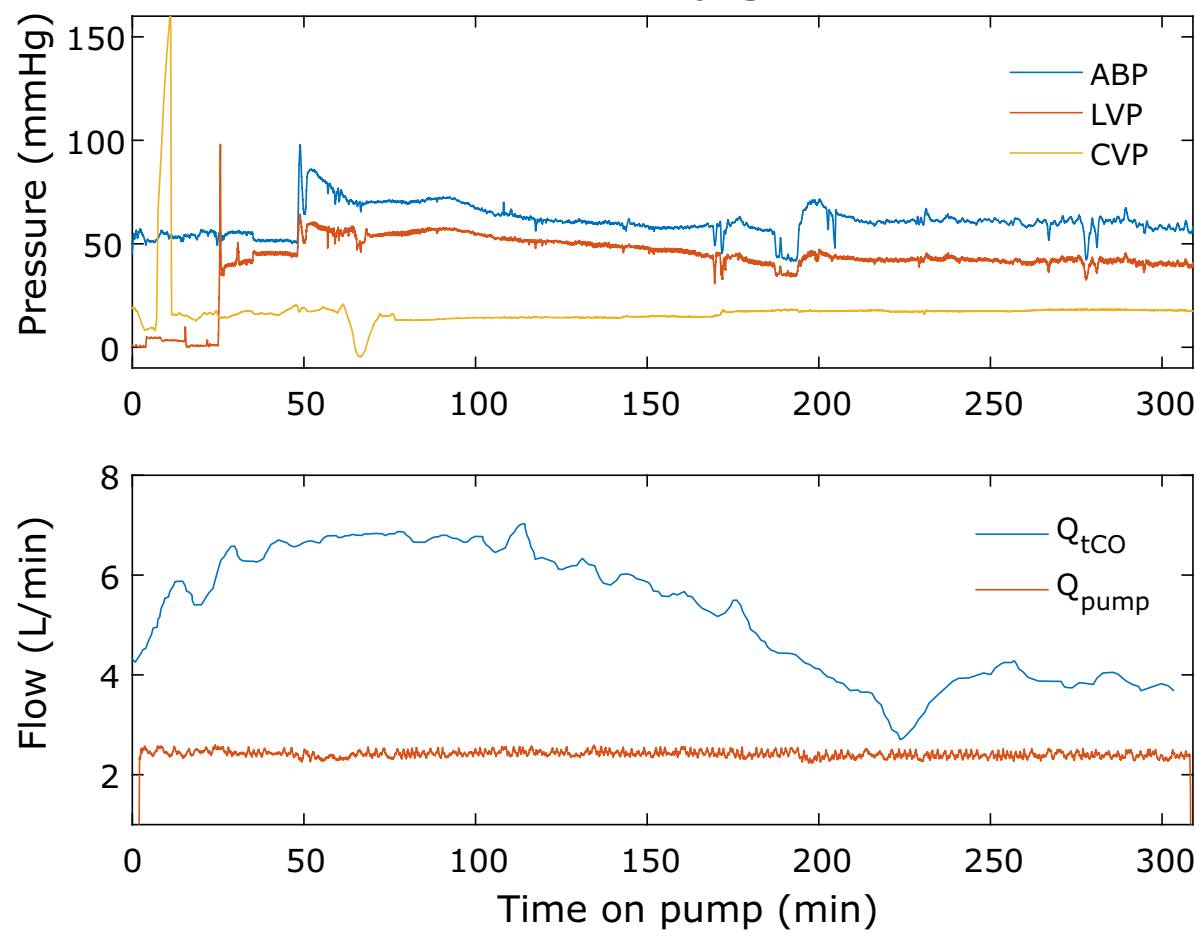

(b)

Animal 4
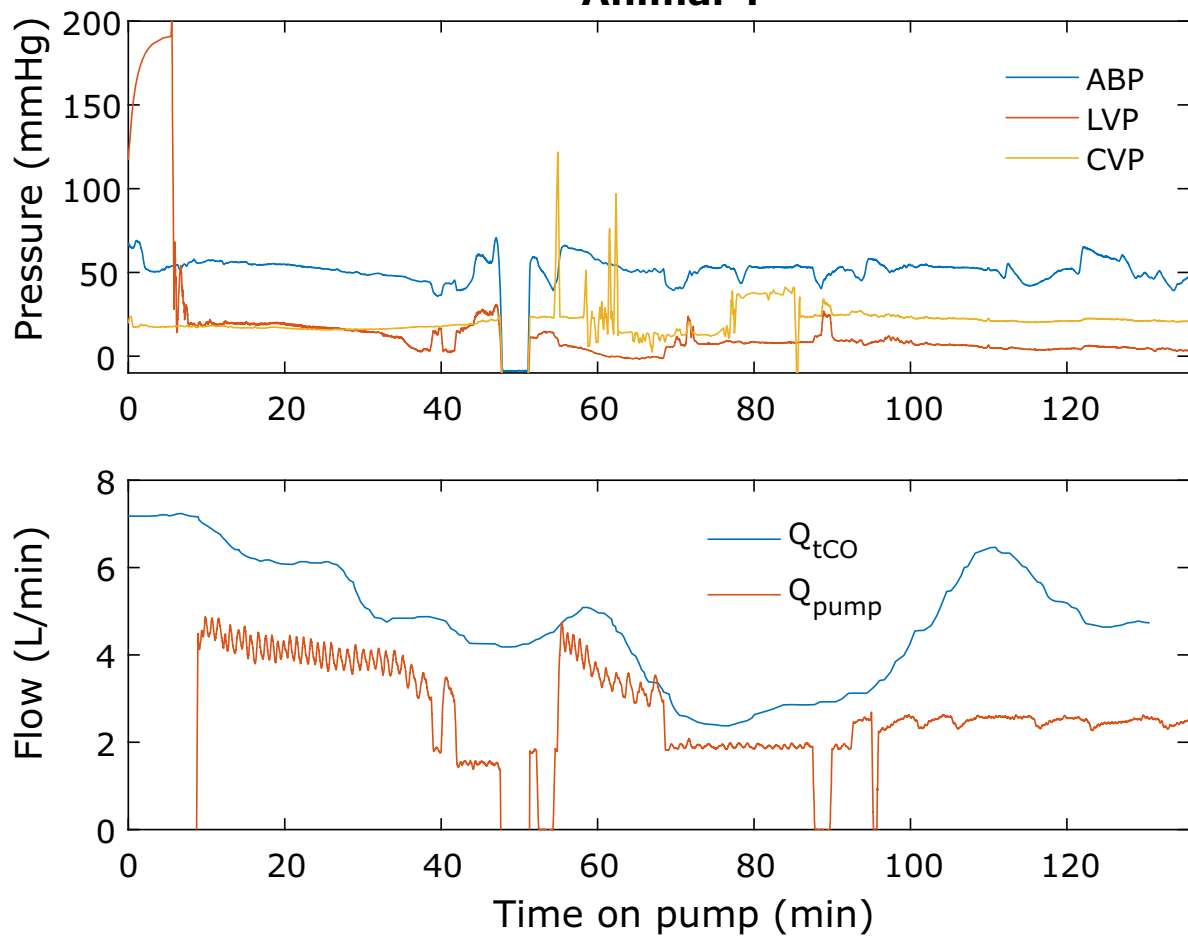

FIGURE 7. Prevailing pressures and flows during pump operation. The top panels of (a) and (b) show the arterial blood pressure (ABP), left ventricular pressure (LVP) and central venous pressure (CVP) of animal 3 and 4, respectively. The bottom panels of (a) and (b) show the blood flow through the pump $\left(Q_{\text {pump }}\right)$ and total cardiac output $\left(Q_{t c o}\right)$ consisting of blood flow through the pump and aortic valve combined. 


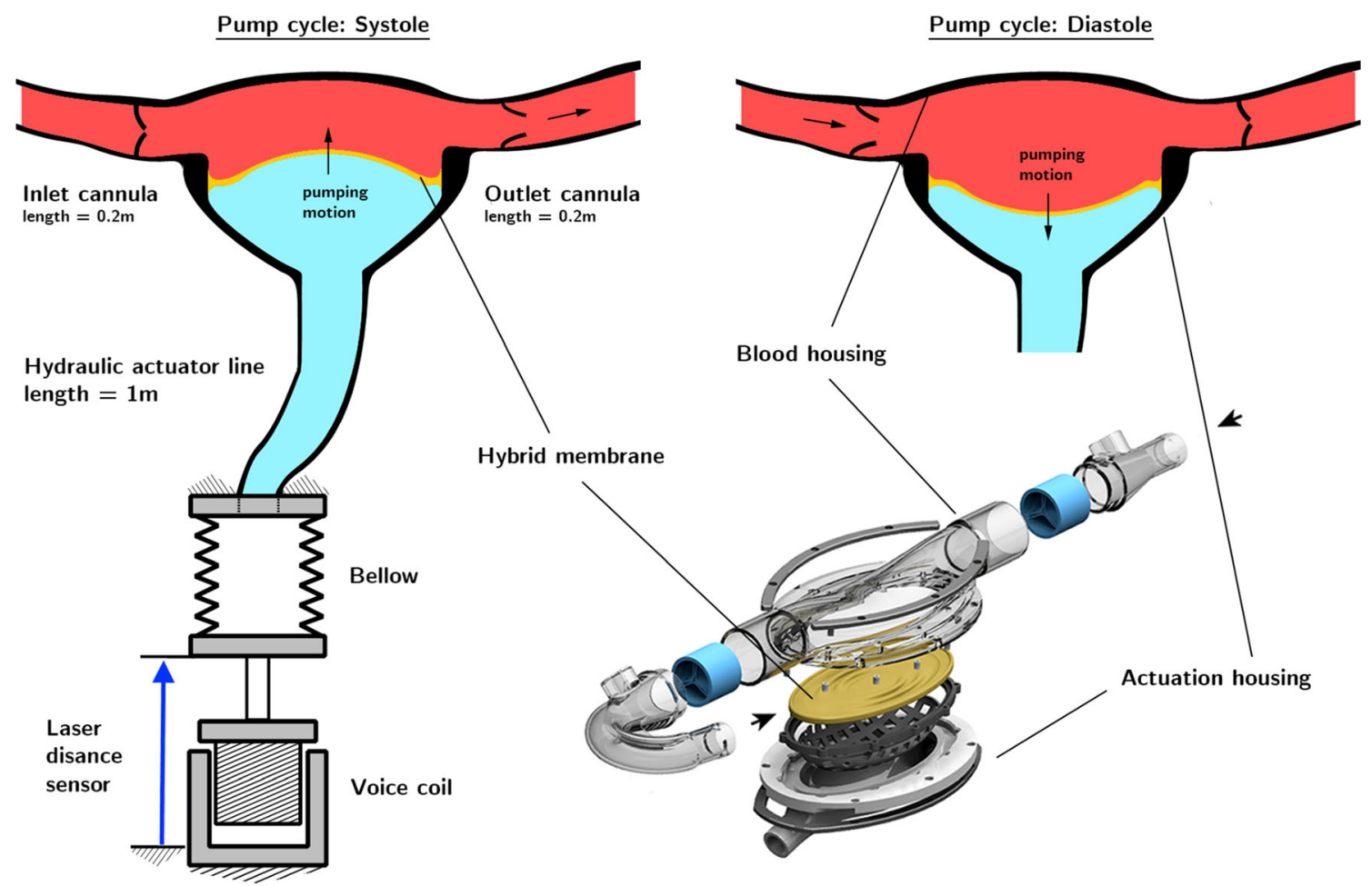

FIGURE 8. Schematic of pump actuation unit consisting of the HyMem-VAD at the top, a hydraulic line connecting the HyMemVAD to the bellows, a PTFE bellows and a voice coil that is used to deform the bellows.

ized and actuated, therefore representing the full experimental result of the in vivo tests. Complete details on the actuation parameters in these animals are provided in Table 1.

\section{End-Point Analysis}

At the end of each experiment, the HyMem-VAD was cross clamped and carefully removed from the animal body. The actuation lid and the hybrid membrane were dismounted and carefully rinsed with warm medium to clear the surface from blood and preserve the sample. The membranes were then immediately fixed.

To evaluate the performance of the endothelialization procedure and thus the maintenance of a connected endothelial tissue, the fixed specimens were immunostained to reveal the cell nuclei, the actin cytoskeleton and the cell-to-cell junctions (with $\beta$-catenin; Fig. 5). All tested experimental configurations (i.e., no actuation, actuation at 2 or $4 \mathrm{~Hz}$ ) proved viable for ECs. In fact, the retrieved endothelia were completely intact, ensuring a full coverage of the target substrate with no visible discontinuity. The junctional localization of $\beta$-catenin confirmed full integrity of the monolayer, which was in all comparable to those obtained in control static conditions (Fig. 5d). Quan- titative measurements further showed no significant changes in local cell density upon implant deployment (Fig. 5e).

\section{DISCUSSION}

The presented study demonstrates that the HyMemVAD implantation protocol and actuation schemes are conducive to device endothelialization, preserving a living endothelium for the entire test duration. Additionally, the endothelialization procedure was successfully adapted to the clinical settings. Altogether, the results demonstrate the feasibility of the innovative concept and represent the necessary basis for its further development. Future validation and translational work will require to secure a complete protection to the implant materials getting in contact with blood. For this to be achieved, we envision a fabrication process that implements the selected topography on the entire luminal surface. The procedure shall be applied to multiple non-planar material interfaces (elastomers, metals, and plastic polymers) therefore requiring optimization of the fabrication protocol. This additional effort will base on the feasibility study presented and will establish the all-round technology for preclinical tests, which shall be performed with a fully 
protected device and tubing. In fact, any bare surface in contact with blood would represent a site for the initiation of thrombotic events, therefore posing a threat to the success of the entire new concept.

\section{The Hyperelastic Membrane Design and Validation}

The hyperelastic membrane represents the technological core of the HyMem-VAD concept (Fig. 1). The design allows a cyclic membrane actuation up to $4 \mathrm{~Hz}$ while avoiding the generation of excessive strains at the luminal surface (Figs. 2 and 3). This results in pumping rate of up to $5 \mathrm{~L} / \mathrm{min}$ and qualifies the HyMem-VAD as potential support for end stage heart failure patients ${ }^{13,15}$ and device endothelialization (Fig. 7). The choice of operation at $4 \mathrm{~Hz}$ is a limitation of the current design, which however allows the containment of the overall pump dimensions. The aortic pulsatility was not in the scope of this study and no sensor was placed in the vicinity of the aorta. Two separate studies have investigated pulsatile VADs operated at frequencies that exceed the heart rate. ${ }^{29,30}$ The in silico and in vitro results demonstrated that pulsatility remains sufficiently high despite the high frequency of operation. In all, the present work is a unique example of a converging approach in which engineering and biological requirements are considered together during the design phase. It demonstrates that physiologicallyrelevant actuation and device endothelialization are possible and compatible in a heart failure support device (Fig. 8).

The current pump design and overall size are optimized towards its intended experimental use and not to achieve implantability. The prototype features a large flange, clamping rings and screw connections to clamp the two halves of the pump housing to facilitate mounting and demounting of membranes and valves. Additionally, the cannulas are designed for extracorporeal animal experiments and specifically to visually assess the state of the pump. For this purpose the outlet cannula features a big curvature as shown in Fig. 1.

Future development for clinical translation will consider a more compact design. In particular, for a clinically-optimized prototype such design elements (flange, clamping rings and screw connections) will be eliminated and the residual volume conformed by the actuation. The blood chamber with connections for the cannulas will be similarly reduced in size. In parallel, we envision a new design of the cannulas and pump body to achieve an implantation configuration with curved inlet cannula and straight outlet. The reduction of the pump size through the incorporation of increased stroke frequencies is instrumental in this approach.

\section{The In Vitro Endothelialization Approach}

The endothelialization of artificial substrates has been applied to attain in vivo protection of vascular grafts. ${ }^{6}$ In this case, successful coverage was based on the in vitro generation of a precast autologous endothelium. ${ }^{41}$ Alternatively, porous materials have been applied to foster efficient transmural ingrowth. ${ }^{26}$ More recently, attempts to extend this procedure to vascular stents exploited the in vivo regenerative potential of circulating endothelial precursors, locally recruited to the luminal interface. ${ }^{25,28}$ VAD endothelialization involves tissue generation and maintenance under complex flow conditions ${ }^{36}$ which can be locally mitigated by the implementation of surface topographies interfering with flow. ${ }^{4}$

The here-reported tests demonstrate that a rationally-designed honeycomb topography (Fig. 1) can support the generation and maintenance of a fullyconnected and functional endothelium (Figs. 4 and 5) in representative regions of the HyMem-VAD featuring a dynamically changing hemodynamic landscape. ${ }^{18,20}$ The same fabrication approach can be extended to other regions of the system. In addition, the precast endothelium was generated using cells isolated from the Vena Saphena, which represents a potential source of endothelialization for cardiovascular devices in human patients. Based on these considerations, the endothelialization protocol can theoretically be upscaled to cover the entire luminal surface of the HyMem-VAD.

From the biological point of view, we have obtained a sound proof that the chosen materials, the selected interface topography, the endothelialization protocols, the implantation procedure, and the actuation in vivo are all compatible with the survival of a reconstituted endothelium. Endothelial cells and tissues are very delicate and would immediately suffer from non-viable conditions. Therefore, the maintenance of tissue integrity after several hours of actuation is a non-trivial technical challenge and undoubtedly provides a proof of viability on which we can leverage for our future developments. Altogether, these results demonstrate that the HyMem-VAD is compatible with device endothelialization based on the in vitro generation of a precast endothelium. Alternative endothelialization mechanisms, including the contribution of circulating endothelial precursors, may play an additional supportive role.

The response of the endothelium, and of the entire cardiovascular system, upon exposure to high frequency pulsations for extended periods of time will be addressed by the future development of our novel concept. This investigation shall be pursued by means of advanced in vitro bioreactors, enabling the long- 
term actuation of the HyMem-VAD, and chronic animal studies. Altogether, these future efforts shall provide a clear evaluation of the proposed trade-off between low WSS and high actuation frequency.

\section{Limitations}

The present study has several limitations. All reported results were obtained in acute in vivo tests during which the animals were constantly under full anesthesia. Therefore, our conclusions are restricted to the validation of the clinical procedure for pump endothelialization, assembly, implantation and initial actuation. Further chronic experiments will be necessary to evaluate the survival of the endothelium and its long-term adaptation to the pump function in vivo. In addition, the extended time necessary for the preparation and implantation of the pump system contributed to define, and limit, the duration of the actuation phase. The limited time window during which the experimental animals showed stable physiological parameters defined the duration of the actual experiments. The luminal endothelialization of the HyMem-VAD was restricted to a central portion of the hyperelastic hybrid membrane. This was selected due to the prevailing values of hemodynamic stresses to which cells are exposed during pump actuation. While $5 \mathrm{~L} / \mathrm{min}$ are considered a relevant level of support for clinical application, extension to $8-10 \mathrm{~L} /$ min flow represent a desirable feature for a wider applicability of the heart pump. Correspondingly, our future experiments will investigate the feasibility of device actuation and cell viability under increased level of flow.

\section{ELECTRONIC SUPPLEMENTARY MATERIAL}

The online version of this article (https://doi.org/10. 1007/s10439-020-02590-1) contains supplementary material, which is available to authorized users.

\section{ACKNOWLEDGMENTS}

The authors thankfully acknowledge the financial support by the Baugarten Foundation, the Georg und Bertha Schwyzer-Winiker Foundation, the IMG Foundation, the Mäxi Foundation, the Propter Homines Foundation, the Stavros Niarchos Foundation (SNF), and the Uniscientia Foundation as well as the ETH Zurich Foundation and the UZH Foundation. This work is part of the Zurich Heart project under the umbrella of University Medicine Zurich.

\section{AUTHOR CONTRIBUTION}

AF: devised the project and the main conceptual ideas for pump design and endothelialization strategy. CG: worked out all experimental details of cell isolation and purification and contributed to the preparation of the manuscript. BB: was involved in planning and supervising the work and in the fabrication of surface topographies. LB: was involved in the analysis of animal experiments. DB: was involved in the fabrication of valves. PE: devised the project and the main conceptual ideas for pump design and supervised the modelling analysis. RH: worked out all experimental details of hybrid membrane fabrication and testing and contributed to the preparation of the manuscript. SK: supported cell isolation and purification. GK: supervised the modelling analysis. CL: performed the modelling analysis. VM: contributed to all stages of pump preparation for the animal experiments. MM: was involved in planning and supervising the work and the development of control algorithms. GP: supported cell isolation and purification. DP: devised the project and the main conceptual ideas for pump design and endothelialization strategy. MR: worked out all experimental details of pump actuation and control and contributed to the preparation of the manuscript. MSD: was involved in planning and supervising the pump development, the animal experiments and the analysis of pump control and actuation. TS: supported the preparation of animal tests. CS: supervised and performed the animal surgeries. GS: was involved in planning and supervising the animal experiments and in the fabrication of surface topographies. SHS: supervised and performed the animal surgeries. BT: was involved in planning the main conceptual ideas and supervising the valve fabrication. PZ: was involved in planning the fabrication of valves. EP: contributed to the writing of the manuscript. VF: devised the project and the main conceptual ideas for pump design and endothelialization strategy. EM: devised the project and the main conceptual ideas for pump design and endothelialization strategy. All authors discussed the results, contributed to the writing and/or commented the manuscript.

\section{FUNDING}

Open access funding provided by Swiss Federal Institute of Technology Zurich.

\section{CONFLICT OF INTEREST}

Peter Zilla and Deon Bezuidenhhout are directors of Strait Access Technologies, Pty, Ltd. A patent application relevant to the work has been filed. The patent is 
pending: Ferrari, Aldo; Bachmann, Björn; Stefopoulos, Georgios; Bernardi, Laura; Giampietro, Costanza; Poulikakos, Dimos; Hopf, Raoul. 'Topologically structured elastomeric membranes in particular for use in ventricular assist devices'. European Patent Application No. 17201984.6 - 1115. All other authors have nothing to disclose.

\section{OPEN ACCESS}

This article is licensed under a Creative Commons Attribution 4.0 International License, which permits use, sharing, adaptation, distribution and reproduction in any medium or format, as long as you give appropriate credit to the original author(s) and the source, provide a link to the Creative Commons licence, and indicate if changes were made. The images or other third party material in this article are included in the article's Creative Commons licence, unless indicated otherwise in a credit line to the material. If material is not included in the article's Creative Commons licence and your intended use is not permitted by statutory regulation or exceeds the permitted use, you will need to obtain permission directly from the copyright holder. To view a copy of this licence, visit http://crea tivecommons.org/licenses/by/4.0/.

\section{REFERENCES}

${ }^{1}$ Al-Azawy, M. G., A. Turan, and A. Revell. Assessment of turbulence models for pulsatile flow inside a heart pump. Comput. Methods Biomech. Biomed. Eng. 19:271-285, 2016.

${ }^{2}$ Ambardekar, A. V., and P. M. Buttrick. Reverse remodeling with left ventricular assist devices: a review of clinical, cellular, and molecular effects. Circ. Heart Fail. 4:224-233, 2011.

${ }^{3}$ Bachmann, B. J., L. Bernardi, C. Loosli, J. Marschewski, M. Perrini, et al. A novel bioreactor system for the assessment of endothelialization on deformable surfaces. Sci. Rep. 6:38861, 2016.

${ }^{4}$ Bachmann, B. J., C. Giampietro, A. Bayram, G. Stefopoulos, C. Michos, et al. Honeycomb-structured metasurfaces for the adaptive nesting of endothelial cells under hemodynamic loads. Biomater. Sci. 6:2726-2737, 2018.

${ }^{5}$ Badimon, J. J., and C. G. Santos-Gallego. Modulatory role of pulsatility on von Willebrand factor: implications for mechanical circulatory support-associated bleeding. J. Am. Coll. Cardiol. 71:2119-2121, 2018.

${ }^{6}$ Baiguera, S., and D. Ribatti. Endothelialization approaches for viable engineered tissues. Angiogenesis 16:1-14, 2013.

${ }^{7}$ Bartoli, C. R., S. Dassanayaka, K. R. Brittian, A. Luckett, $\mathrm{S}$. Sithu, et al. Insights into the mechanism(s) of von Willebrand factor degradation during mechanical circulatory support. J. Thorac. Cardiovasc. Surg. 147:1634-1643, 2014.
${ }^{8}$ Bartoli, C. R., D. J. Restle, D. M. Zhang, M. A. Acker, and P. Atluri. Pathologic von Willebrand factor degradation with a left ventricular assist device occurs via two distinct mechanisms: mechanical demolition and enzymatic cleavage. J. Thorac. Cardiovasc. Surg. 149:281-289, 2015.

${ }^{9}$ Bazzoni, G., and E. Dejana. Endothelial cell-to-cell junctions: molecular organization and role in vascular homeostasis. Physiol. Rev. 84:869-901, 2004.

${ }^{10}$ Bedair, T. M., M. A. ElNaggar, Y. K. Joung, and D. K. Han. Recent advances to accelerate re-endothelialization for vascular stents. J. Tissue Eng. 8:2041731417731546, 2017.

${ }^{11}$ Bernardi, L., R. Hopf, A. Ferrari, A. E. Ehret, and E. Mazza. On the large strain deformation behavior of silicone-based elastomers for biomedical applications. Polym. Test 58:189-198, 2017.

${ }^{12}$ Dasse, K. A., S. D. Chipman, C. N. Sherman, A. H. Levine, and O. H. Frazier. Clinical experience with textured blood contacting surfaces in ventricular assist devices. ASAIO Trans. 33:418-425, 1987.

${ }^{13}$ Haeck, M. L., G. E. Hoogslag, S. F. Rodrigo, D. E. Atsma, R. J. Klautz, et al. Treatment options in end-stage heart failure: where to go from here? Neth. Heart J. 20:167-175, 2012.

${ }^{14}$ Jufri, N. F., A. Mohamedali, A. Avolio, and M. S. Baker. Mechanical stretch: physiological and pathological implications for human vascular endothelial cells. Vasc. Cell 7:8, 2015.

${ }^{15}$ Katz, J. N., S. B. Waters, I. B. Hollis, and P. P. Chang. Advanced therapies for end-stage heart failure. Curr. Cardiol. Rev. 11:63-72, 2015.

${ }^{16}$ Lampugnani, M. G., M. Corada, P. Andriopoulou, S. Esser, W. Risau, and E. Dejana. Cell confluence regulates tyrosine phosphorylation of adherens junction components in endothelial cells. J. Cell Sci. 110(Pt 17):2065-2077, 1997.

${ }^{17}$ Latremouille, C., A. Carpentier, P. Leprince, J. C. Roussel, B. Cholley, et al. A bioprosthetic total artificial heart for end-stage heart failure: results from a pilot study. J. Heart Lung Transplant. 37:33-37, 2018.

${ }^{18}$ Loosli, C. Numerical and experimental studies for the development of an endothelialized ventricular assist device. ETH dissertation number 26750, 2020.

${ }^{19}$ Loosli, C., L. Moy, G. Kress, E. Mazza, and P. Ermanni. Corrugated diaphragm shape design study for hemocompatible pulsatile ventricular assist devices. Comput. Methods Biomech. Biomed. Eng. 23:1-9, 2018.

${ }^{20}$ Loosli, C., S. Rupp, B. Thamsen, M. Rebholz, G. Kress, et al. High-frequency operation of pulsatile ventricular assist devices: a computational study on circular and elliptically shaped pumps. Int. J. Artif. Organs 37:241-252, 2019.

${ }^{21}$ Mehra, M. R., D. J. Goldstein, N. Uriel, J. C. Cleveland, $\mathrm{Jr}, \mathrm{M}$. Yuzefpolskaya, et al. Two-year outcomes with a magnetically levitated cardiac pump in heart failure. $N$. Engl. J. Med. 378:1386-1395, 2018.

${ }^{22}$ Millan, J., R. J. Cain, N. Reglero-Real, C. Bigarella, B. Marcos-Ramiro, et al. Adherens junctions connect stress fibres between adjacent endothelial cells. BMC Biol. 8:11, 2010.

${ }^{23}$ Nikolaychik, V. V., D. M. Wankowski, M. M. Samet, and P. I. Lelkes. In vitro testing of endothelial cell monolayers under dynamic conditions inside a beating ventricular prosthesis. ASAIO J. 42:M487-M494, 1996.

${ }^{24}$ Ochsner, G., R. Amacher, A. Amstutz, A. Plass, M. Schmid Daners, et al. A novel interface for hybrid mock cir- 
culations to evaluate ventricular assist devices. IEEE Trans. Biomed. Eng. 60:507-516, 2013.

${ }^{25}$ Pang, J. H., Y. Farhatnia, F. Godarzi, A. Tan, J. Rajadas, et al. In situ endothelialization: bioengineering considerations to translation. Small 11:6248-6264, 2015.

${ }^{26}$ Pennel, T., D. Bezuidenhout, J. Koehne, N. H. Davies, and P. Zilla. Transmural capillary ingrowth is essential for confluent vascular graft healing. Acta Biomater. 65:237-247, 2018.

${ }^{27}$ Potapov, E. V., C. Starck, and V. Falk. Regarding The STS/Intermacs 2019 Annual Report. Ann. Thorac. Surg. 2020. https://doi.org/10.1016/j.athoracsur.2019.12.005.

${ }^{28}$ Rafii, S. Circulating endothelial precursors: mystery, reality, and promise. J. Clin. Invest. 105:17-19, 2000.

${ }^{29}$ Rebholz, M., R. Amacher, A. Petrou, M. Meboldt, and M. Schmid Daners. High-frequency operation of a pulsatile VAD: a simulation study. Biomed. Eng-Biomed. Tech. 62:161-170, 2017.

${ }^{30}$ Rebholz, M., S. A. Dual, M. Batliner, M. Meboldt, and M. Schmid Daners. Short-term physiological response to highfrequency-actuated pVAD support. Artif. Organs 43:1170 1181, 2019.

${ }^{31}$ Restle, D. J., D. M. Zhang, G. Hung, J. L. Howard, F. Kallel, et al. Preclinical Models for Translational Investigations of Left Ventricular Assist Device-Associated von Willebrand Factor Degradation. Artif. Organs 39:569-575, 2015.

${ }^{32}$ Rosenberg, G., C. A. Siedlecki, C. S. Jhun, W. J. Weiss, K. Manning, et al. Acquired Von Willebrand Syndrome and Blood Pump Design. Artif. Organs 42:1119-1124, 2018.

${ }^{33}$ Schmitto, J. D., D. Zimpfer, A. E. Fiane, R. Larbalestier, $\mathrm{S}$. Tsui, et al. Long-term support of patients receiving a left ventricular assist device for advanced heart failure: a follow-up analysis of the Registry to Evaluate the HeartWare Left Ventricular Assist System. Eur. J. Cardiothorac. Surg. 50:834-838, 2016.
${ }^{34}$ Scott-Burden, T., C. L. Tock, J. P. Bosely, F. J. Clubb, Jr, S. M. Parnis, et al. Nonthrombogenic, adhesive cellular lining for left ventricular assist devices. Circulation 98:II339-II345, 1998.

${ }^{35}$ Slater, J. P., E. A. Rose, H. R. Levin, O. H. Frazier, J. K. Roberts, et al. Low thromboembolic risk without anticoagulation using advanced-design left ventricular assist devices. Ann. Thorac. Surg. 62:1321-1327, 1996.

${ }^{36}$ Sonntag, S. J., T. A. S. Kaufmann, M. R. Busen, M. Laumen, T. Linde, et al. Simulation of a pulsatile total artificial heart: development of a partitioned Fluid Structure Interaction model. J. Fluid Struct. 38:187-204, 2013.

${ }^{37}$ Teuteberg, J. J., J. C. Cleveland, Jr, J. Cowger, R. S. Higgins, D. J. Goldstein, et al. The Society of Thoracic Surgeons Intermacs 2019 Annual Report: the changing landscape of devices and indications. Ann. Thorac. Surg. 109:649-660, 2020.

${ }^{38}$ Timms, D. A review of clinical ventricular assist devices. Med. Eng. Phys. 33:1041-1047, 2011.

${ }^{39}$ Ton, V. K., R. Xie, J. A. Hernandez-Montfort, B. Meyns, T. Nakatani, et al. Short- and long-term adverse events in patients on temporary circulatory support before durable ventricular assist device: an IMACS registry analysis. $J$. Heart Lung Transplant. 39:342-352, 2020.

${ }^{40}$ Vincent, F., A. Rauch, V. Loobuyck, E. Robin, C. Nix, et al. Arterial pulsatility and circulating von Willebrand factor in patients on mechanical circulatory support. J. Am. Coll. Cardiol. 71:2106-2118, 2018.

${ }^{41}$ Zilla, P., U. Vonoppell, and M. Deutsch. The endothelium: a key to the future. J. Cardiac Surg. 8:32-60, 1993.

Publisher's Note Springer Nature remains neutral with regard to jurisdictional claims in published maps and institutional affiliations. 